\title{
Manybody treatment of white dwarf and neutron stars on the brane
}

\author{
Mofazzal Azam ${ }^{1}$ and M. Sami ${ }^{2}$ \\ ${ }^{1}$ Theoretical Physics Division, \\ Bhabha Atomic Research Centre \\ Mumbai, India \\ ${ }^{2}$ Inter-University Centre for Astronomy and Astrophysics \\ Pune, India
}

\begin{abstract}
Brane-World models suggest modification of Newton's law of gravity on the 3-brane at submillimeter scales. The brane-world induced corrections are in higher powers of inverse distance and appear as additional terms with the Newtonian potential. The average inter-particle distance in white dwarf and neutron stars are $10^{-10} \mathrm{cms}$ and $10^{-13} \mathrm{cms}$ respectively, and therefore, the effect of submillimeter corrections needs to be investigated. We show, by carrying out simple manybody calculations, that the mass and mass-radius relationship of the white dwarf and neutron stars are not effected by submillimeter corrections. However, our analysis shows that the correction terms in the effective theory give rise to force akin to surface tension in normal liquids.
\end{abstract}

PACS numbers:

\section{INTRODUCTION}

String theory is considered as a strong candidate for the unified theory of the standard model and gravity. However, string theory is a higher dimensional theory, and therefore, it is necessary to find a mechanism to get rid of the extra dimensions with some imprint left on the four dimensional physical world. But such effects must be consistent with the existing experimental and observational data. The brane-world scenario proposed by Randall and Sundrum [1, 2] for describing a low dimensional physical theory starting from a higher dimensional theory has attracted lot of attention recently $[\underline{3}$. The model has triggered a large amount of work in cosmology and astrophysics [4].

The brane-world scenario leads to modification of gravity at submillimeter distance scales. The brane corrections are in higher powers of inverse distance and appear as additional terms in the Newtonian potential. In the RandallSundrum scenario, the modification suggested for the Newtonian potential is given by,

$$
\Phi(r)=\frac{G M}{r}\left(1+\frac{2 l^{2}}{3 r^{2}}\right)
$$

where $l$ is the curvature scale of 5 -dimensional anti-deSitter space time, and $G$ is the usual Newton's constant of gravity, $M$ is the mass, $r$ is the distance in 3 -space. In the setting above, $l$ has the dimension of length in 3 -space, in other words, it is measured in centimeters in standard CGS units. Henceforth, we will take $l_{s}^{2}=\frac{2}{3} l^{2}$

Several groups have estimated bounds on the distance scales at which such corrections may be allowed without violating the existing experimental and observational data [8]. As mentioned above, there have also been some works dealing with the astrophysical consequences of such modifications of gravity [5, 6, 7]. The average inter-particle distance in white dwarf and neutron stars are $10^{-10} \mathrm{cms}$ and $10^{-13} \mathrm{cms}$ respectively, and therefore, the effect of submillimeter corrections needs to be investigated. Wiseman [7] has carried out a detailed numerical study of the neutron stars on the brane and claimed that the normal parameters of the neutron stars such as mass and radius are not effected by brane world corrections. In this paper, we carry out simple manybody analytical calculations using the brane modified gravitational potential. Our approach is based on manybody theory in semi-relativistic quantum mechanics [9, 10]. At first, we briefly summerise the nature of the potential and the force that emerges in the manybody context due to the barneworld correction to gravity (the detail calculation is presented in appendix-I). We find that, for extended massive bodies, the nature of the force and the potential due to brane correction terms is very different from those due to Newtonian gravity. For example, inside a hollow spherical shell the potential due to Newtonian gravity is constant, independent of location of the test particle. Therefore, the force is zero inside the shell. We show that this is not true in the presence of the brane correction term. This result may have some relevance in context of experimental observation of deviations from Newton's law of gravity. We also show that the brane-world correction to gravity gives rise to force akin to surface tension in normal liquids. Following that we consider the effects due to the brane-world corrections on the mass and the mass-radius relationship of white dwarf and neutron stars. We find that the brane world corrections do not influence these two parameters in any significant way.

In the appendix-I, we provide detailed calculations of the potential, potential energy and the force due to the brane correction term. We also point out that many new physical features that emerge from corrections to Newtonian gravity are generic due to the deviation from the inverse square law of force and does not depend on the particular 
form of the correction term.

In appendix-II, we analyse the nature of the extrema of various energy functionals that appear in the text.

\section{MANY-BODY EFFECTS OF BRANE WORLD CORRECTION TO GRAVITY}

We consider in this section the many-body effects of brane world correction to gravity in a classical theory. Essentially, we carry out analysis of the potential and force due to brane world correction for spherically symmetric body of constant density and a fixed radius. Details of calculations are given in appendix-I. Here we provide a brief summary of these results. As already mentioned in the introductory section, for extended massive bodies, the nature of the force and the potential due to brane correction terms is very different from those due to Newtonian gravity. These claims are demonstrated latter in this section.

For a spherically symmetric ball of constant density $\rho$ and radius $a$, the potential due to brane corrections at a point $\vec{r}(r>a, r$ being the distance from centre of the ball) outside the ball is given by,

$$
\Phi_{B}(r)=-2 \pi G l_{s}^{2} \rho\left[\ln \frac{r+a}{r-a}-\frac{2 a}{r}\right]
$$

The corresponding force is given by,

$$
F=2 \pi G l_{s}^{2} \rho\left[\frac{1}{r+a}-\frac{1}{r-a}+\frac{2 a}{r^{2}}\right]=-4 \pi a G l_{s}^{2} \rho\left[\frac{1}{r^{2}-a^{2}}-\frac{1}{r^{2}}\right]
$$

When the point $\vec{r}$ is close to surface of the ball, both potential and the force grows and becomes very large.Therefore, one needs to introduce a parameter $\epsilon$ which characterises the smallest inter-particle distance. At a distance $\epsilon$ from the surface of the ball, the force is given by,

$$
F=2 \pi G l_{s}^{2} \rho\left[-\frac{1}{\epsilon}+\frac{2}{a}\right]
$$

This is clearly a skin effect. However, this force is not as strong as one may think. At a distance of $1 \mathrm{~mm}$ from the surface of a neutron star, this force is weaker than the force of Newtonian gravity. The is because this force depends on the density and not on the mass. However, this force can be large close to the surface of a spherical grain of very small radius and may even overtake the force due to Newtonian gravity.

For a spherically symmetric shell of outer radius $R$ and inner radius $b$, the potential at an interior point $\vec{r}(r<b)$ is given by,

$$
\Phi_{B}^{\prime}(r)=-2 \pi G l_{s}^{2} \rho \ln \frac{R^{2}-r^{2}}{b^{2}-r^{2}}
$$

Force due to this potential, at distance $r$ from the centre is given by,

$$
F(r)=2 \pi G l_{s}^{2} \rho\left[\frac{2 r}{b^{2}-r^{2}}-\frac{2 r}{R^{2}-r^{2}}\right]
$$

From the expression above it is obvious that $F(r)>0$. This means that the force is directed in the direction in which $r$ increases. In other words, it is directed towards the inner boundary surface of the shell. At the centre of the shell, $r=0$, and therefore, the force is zero. But any small perturbation will move the test particle away from the centre towards inner boundary surface. This is a new element that emerges from the brane-world correction to Newton's law of gravity. We know that the force due to Newtonian gravity is zero inside a spherically symmetric shell. If one can find the above mentioned effect inside a shell, it will provide a very clean test of brane world corrections to Newtonian gravity. May be such experiments can be carried out on board artificial satellites.

In this case, as in the case of spherical ball, there is skin effect. The force of attraction close to the inner boundary of the shell at distance, say, $\epsilon$ from the inner boundary surface is given by,

$$
F=2 \pi G l_{s}^{2} \rho\left[\frac{1}{\epsilon}-\frac{2 b}{R^{2}-r^{2}}\right]
$$

Therefore, any interior layer of a spherical ball experiences two types of forces: one is due to a sphere just bellow it and other is due to the spherical shell outside. These two types of forces, individually, give rise to a large forces on this layer due to the skin effect. Remarkably, these two forces act in opposite directions and the forces due to 
skin effect are equal in magnitude and exactly cancel each other. On the layer at $r$, let $F_{1}$ be the force due the inner sphere and $F_{2}$ be the force due to the outer shell.

$$
\begin{gathered}
F_{1}=2 \pi G l_{s}^{2} \rho\left[-\frac{1}{\epsilon}+\frac{2}{r}\right] \\
F_{2}=2 \pi G l_{s}^{2} \rho\left[\frac{1}{\epsilon}-\frac{2 r}{R^{2}-r^{2}}\right]
\end{gathered}
$$

Therefore, the net force at any point on the layer at a distance $r$ from the centre of the ball is given,

$$
F=F_{1}+F_{2}=2 \pi G l_{s}^{2} \rho\left[\frac{2}{r}-\frac{2 r}{R^{2}-r^{2}}\right]
$$

This result is valid for every layer except the one at the outer most boundary. For the outer most layer, there is an inner sphere but no outer shell. There is no cancellation of the skin effect on this layer. Therefore, it experiences a large force.This will give rise to effect similar to surface tension in normal liquids.

The formula above also shows that the net force due to the brane-world induced corrections at any point on layers at distance $r$ where $r<\frac{R}{\sqrt{2}}$ is repulsive, and on layers at a distance $r, r>\frac{R}{\sqrt{2}}$ is attractive. Within a submillimeter central core of a neutron star there are approximately $10^{36}$ neutrons arranged in approximately $10^{12}$ layers. At any point on these layers the net repulsive force due to the brane-world induced corrections overtake the attractive force due to Newtonian gravity. Also note that any change in the radius of the star due to mass acreation or mass loss leads to the rearrangement of the field of force in the interior of the star. Therefore, the interior and the exterior of the relativistic stars are intimately (although very weakly) connected to each other in the presence of brane-world corrections.

The potential energy of a spherically symmetric body (of constant density) of mass $M$ and radius $R$, due to the brane-world induced corrections is given by,

$$
U=-\frac{3 G M^{2} l_{s}^{2}}{R^{3}} \ln \frac{R}{\epsilon}=-\frac{3 G N^{2} m^{2} l_{s}^{2}}{R^{3}} \ln \frac{R}{\epsilon}
$$

Here $N$ is the number of particles in the spherical ball of radius $R, m$ is the mass of the elementary constituent particles, In a classical theory, the parameter $\epsilon$ should be considered as the minimum inter-particle distance. But in a quantum theory, as we will show in the latter section, it is possible to fix its' value using Heisenberg's uncertainity relation and Pauli's exclusion principle.

\section{MASS-RADIUS RELATIONSHIP OF THE WHITE DWARF AND NEUTRON STARS ON THE BRANE}

In this section, we show that the brane world corrections to Newton's law of gravity does not influence the mass-radius relationship of white dwarf and neutron statrs in any significant way. Let us consider a system of large number of fermions contained in some volume $V$. Let us also assume that the system is almost a compact sphere with a large constant density such that fermions are relativistic. We will consider both the white dwarf and neutron stars. We do not discuss here the details of the nuclei present in the white dwarf stars. We simply consider that, in the white dwarf stars, there are two types of particles: the electrons and the nucleons. For the nucleons, we have in mind the protons and the neutrons. The over all charge neutrality of the star implies that number of electrons and the protons should be equal. This suggests that for $N$ electrons in the star there should be $2 N$ nucleons ( $N$ protons and $N$ neutrons). However, we will consider that the number of electrons is equal to the number of nucleons which are essentially protons. Such a choice changes the mass and radius of the white dwarf star by a small numerical factor but the analysis is easily carried over to the case of neutron stars. The zero-point kinetic energy in the white dwarfs is solely due to the zero-point motion of the electrons but the gravitational potential energy is soley due to the gravitational interaction of the nucleons among themselves. For the neutron stars the kinetic as well as potential energy is generated by the neutrons alone. We assume that the mass of a nucleons is equal to the mass of a neutron. We also consider a situation when the kinetic energy due to temperature is much small compared to the zero point kinetic energy and, therefore, can be neglected.

To make our derivation more transparent, at first, we derive the mass-radius relationship without the brane correction terms. Let us consider first the white dwarfs. We have a spherically symmetric region of radius $R$ containing $N$ electrons and $N$ nucleons. We assume the electrons to be relativistic; their total kinetic energy is,

$$
K_{N}=N c\left(p^{2}+m_{e}^{2} c^{2}\right)^{1 / 2}-N m_{e} c^{2}
$$


Substituting the value of zero-point momentum, $p=\frac{\hbar N^{1 / 3}}{R}$ in the expression of the kinetic energy, we obtain the total energy as

$$
E(R)==N c\left(\frac{\hbar^{2} N^{2 / 3}}{R^{2}}+m_{e}^{2} c^{2}\right)^{1 / 2}-N m_{e} c^{2}-\frac{3}{5} \times \frac{G m_{n}^{2} N^{2}}{R}
$$

The quantum mechanical variational ground state is obtained by demanding, $\frac{d E(R)}{d R}=0$.This gives,

$$
\frac{N^{1 / 3} \hbar}{\left(\hbar^{2} N^{2 / 3}+m_{e}^{2} c^{2} R^{2}\right)^{1 / 2}}=\frac{3}{5} \times \frac{G m_{n}^{2} N^{2 / 3}}{c \hbar}
$$

Both the LHS and RHS of the equation above are positive. The LHS, as is clear from equation above, is strictly less than one, and therefore, the RHS is strictly less than one.In particular

$$
\frac{3}{5} \times \frac{G m_{n}^{2} N^{2 / 3}}{c \hbar}<1
$$

This provides an upper bound on the number of particles and therefore, an upper bound on the mass of the star. As before, the upper bound on the number of particles, $N_{\max }$, is given by,

$$
N_{\max }^{2 / 3}=\frac{5}{3} \times \frac{c \hbar}{G m_{n}^{2}}
$$

Therefore, as before the upper bound on the mass of the star is $M_{\max }=m_{n} N_{\max }$. Let us call the radius defined as,

$$
R_{0}=\frac{\hbar N^{1 / 3}}{m_{e} c}
$$

Compton radius of a compact relativistic white dwarf. Let us also introduce the notation,

$$
\alpha=\left(N / N_{\max }\right)^{2 / 3}
$$

Using this, we can write Eq.(19) as

$$
\frac{1}{\left(1+\frac{R^{2}}{R_{0}^{2}}\right)^{1 / 2}}=\alpha
$$

This can also be expressed as,

$$
R=R_{0} \sqrt{\frac{1}{\alpha^{2}}-1}
$$

This is the mass-radius relationship of white dwarfs in the absence of brane correction term. (It is shown in appendix-II, that this solution corresponds to the minimum of the total energy, as a function of $R$ ). Note that $\alpha$, by definition, is strictly less than one. We can get another bound on $\alpha$ as follows. The white dwarf star that we are considering is relativistic, and therefore, $p=\frac{\hbar N^{1 / 3}}{R} \geq m_{e} c$. This implies that $R \leq \frac{\hbar N^{1 / 3}}{m_{e} c}=R_{0}$. Taking $R \leq R_{0}$, in the equation above, we find that $\alpha \geq \frac{1}{\sqrt{2}}$. An order of magnitude calculation gives the mass of the white dwarf between one to two solar masses and the radius a few thousand kilometers. Neutron star parameters are obtained simply by replacing the electron mass by neutron mass. As an order of magnitude estimate, the mass of the neutron stars turns out to be similar to the white dwarf mass but the radius is just a few kilometers.

Let us now consider the effect of the brane-world induced correction term on the parameters of white dwarf and the neutron stars.

The potential energy of spherically symmetric ball due to Randall-Sundrum brane corrections is given by,

$$
U_{R S}=-\frac{3 G l s^{2} m_{n}^{2} N^{2}}{R^{3}} \times \ln \frac{R}{\epsilon}
$$

Therefore, the total energy including brane correction is given by,

$$
E(R)=K_{N}+U_{N}(R)+U_{R S}=N c\left(p^{2}+m_{e}^{2} c^{2}\right)^{1 / 2}-N m_{e} c^{2}-\frac{3}{5} \times \frac{G m_{n}^{2} N^{2}}{R}-\frac{3 G l s^{2} m_{n}^{2} N^{2}}{R^{3}} \times \ln \frac{R}{\epsilon}
$$


Substituting $p=\frac{\hbar N^{1 / 3}}{R}$, we obtain

$$
E(R)=N c\left(\frac{\hbar^{2} N^{2 / 3}}{R^{2}}+m_{e}^{2} c^{2}\right)^{1 / 2}-N m_{e} c^{2}-\frac{3}{5} \times \frac{G m_{n}^{2} N^{2}}{R}-\frac{3 G l s^{2} m_{n}^{2} N^{2}}{R^{3}} \times \ln \frac{R}{\epsilon}
$$

The quantum mechanical variational ground state is obtained as before,

$$
\frac{N^{1 / 3} \hbar}{\left(\hbar^{2} N^{2 / 3}+m_{e}^{2} c^{2} R^{2}\right)^{1 / 2}}=\frac{3}{5} \times \frac{G m_{n}^{2} N^{2 / 3}}{c \hbar}+\frac{9 G l s^{2} m_{n}^{2} N^{2 / 3}}{R^{2} c \hbar} \times \ln \frac{R}{\epsilon}
$$

Note that $\epsilon$ was introduced as shortest inter layer distance in the spherical ball. From physics point of view it can be identified with the shortest inter-particle distance. For fermions, as per Pauli's exclusion principle, it can not be shorter than $R / N^{1 / 3}$. We take $\epsilon=R / N^{1 / 3}\left(\ln \frac{R}{\epsilon}=\ln N^{1 / 3}\right)$.

Both the LHS and RHS of the equation above are positive. The LHS, as is clear from equation above, is strictly less than one, and therefore, the RHS is strictly less than one.In particular

$$
\frac{3}{5} \times \frac{G m_{n}^{2} N^{2 / 3}}{c \hbar}<1
$$

This provides an upper bound on the number of particles $N$, and therefore, an upper bound on the mass of the star, $M=m_{n} N$. We will proceed in the same way as we did in the absence of the brane correction terms. However, in this case we obtain a cubic equation for the square of the radius. Before we solve the equation, let us introduce the following notation,

$$
\beta=\frac{15 l s^{2}}{R_{0}^{2}} \times \ln \frac{R}{\epsilon}=\frac{15 l s^{2}}{R_{0}^{2}} \times \ln N^{1 / 3}
$$

We introduce a new variable,

$$
Y=\left(\frac{R}{R_{0}}\right)^{2}
$$

The equation Eq.(15), in terms of the new variable can be written as,

$$
\frac{1}{(1+Y)^{1 / 2}}=\alpha+\frac{\alpha \beta}{Y}
$$

After some algebraic manipulations, we obtain

$$
Y^{3}+\left(2 \beta+1-\frac{1}{\alpha^{2}}\right) Y^{2}+\left(2 \beta+\beta^{2}\right) Y+\beta^{2}=0
$$

Before we look for solution of this equation, let us have, an order of magnitude, estimate of the values of parameters $\alpha$ and $\beta$. They are both pure numbers. As mentioned earlier, both for white dwarf and neutron stars, the range of $\alpha$ is given by $\frac{1}{\sqrt{2}} \leq \alpha<1$. The parameter $\beta$, depends on the Compton radius of the star, and therefore, its' value is different for white dwarf and the neutron stars. Taking $N \approx 10^{57}$, we find that for white dwarfs, $\beta \approx 10^{-16}$, and for neutron stars, $\beta \approx 10^{-10}$. The real root of Eq.(7) leads to the following,

$$
\frac{R}{R_{0}}=\sqrt{\frac{1}{\alpha^{2}}-1}+\mathcal{O}(\beta)
$$

To make the dependence on the brane world parameter $l_{s}$ explicit, we can also write the equation above as,

$$
\frac{R}{R_{0}}=\sqrt{\frac{1}{\alpha^{2}}-1}+\mathcal{O}\left(\frac{l_{s}^{2}}{R_{0}^{2}}\right)
$$

These equations are essentially same as Eq.(4) because corrections are insignificant. Note that Eq.(7), is a cubic equation, and therefore, there are two more solutions of the equation. Up to order $\beta$, these two solutions are coincident, and are given by

$$
\frac{R}{R_{0}}=\mathcal{O}(\beta)=\mathcal{O}\left(\frac{l_{s}^{2}}{R_{0}^{2}}\right)
$$


It is shown, in appendix-II, that these solutions correspond to the maxima of the total energy as a function of $R$. They clearly do not lead to any stable configuration of the compact stars. As shown in appendix-II, the solution given by Eq.(8), corresponds to the minimum of the total energy as a function of $R$. Therefore, in the effective four dimensional theory, the standard parameters of the white dwarf and neutron stars do not depend on the brane world induced correction term.However, we note that the brane world corrections give rise to surface tension which is absent in Newtonian gravity as well as general theory of relativity. This surface tension is the new element in gravity. However, we still do not know whether it has any observable signature.

\section{CONCLUSIONS}

In this paper we have examined the influence of brane world corrections on the parameters of white dwarfs and neutron stars using manybody theory. The brane corrections to Newtonian potential give rise to interesting new effects, however, these corrections do not influence the stability of the stars in a significant way. For instance, the mass, radius and the mass-radius relation remains unchanged. We have demonstrated these features using a simple analytical treatment. Our results are in qualitative agreement with the numerical work by Wiseman 7].

We also point out many new physical features that emerge from corrections to Newtonian gravity. These physical features are generic due to the deviation from the inverse square law of force and does not depend on the particular form of the correction term: (a.) In the many body context, force as well as potential due to the brane correction term depends on density rather than the mass. Therefore, we expect that the relative strength of the force compared to Newtonian force will be more close to the surface of spherical grains of smaller radius. (b.) Newton-Birkhoff theorem is violated by the brane world induced correction terms. Therefore, inside a hollow spherical shell there will be force. This may turn out to be useful for experimental verification of Randall-Sundrum or any other similar corrections to gravity. (c) Violation of Newton-Birkhoff theorem due to the presence of brane-world correction terms, gives rise to force similar to surface tension in normal liquids.Neither Newtonian gravity nor the general theory of relativity give rise to force akin to surface tension. The correction term shares many similarities with molecular force in normal Liquids. At present, we are not very clear which of the new features discussed here are relevant to observations. In our opinion, this is an important issue which requires further investigation.

\section{ACKNOWLEDGMENTS}

We thank Roy Maartens for his critical comments on the first draft of the manuscript. We also thank N. Dadhich and A. Kembhavi for helpful discussions.

\section{APPENDIX-I}

We want to calculate the gravitational potential energy of a spherically symmetric ball of radius $R$ and constant density $\rho$.Here the two-body gravitational potential energy consists of two parts.First part is the standard Newtonian part given by,

$$
U_{N}=-\frac{G m_{1} m_{2}}{\left|\vec{r}-\overrightarrow{r^{\prime}}\right|}
$$

where $\vec{r}$ and $\overrightarrow{r^{\prime}}$ are the locations of the point masses $m_{1}$ and $m_{2}$ respectively. The Newtonian potential at a point $\vec{r}$ due to a point mass $m$ at point $\overrightarrow{r^{\prime}}$ is given by

$$
\Phi_{N}(\vec{r})=-\frac{G m}{\left|\vec{r}-\overrightarrow{r^{\prime}}\right|}
$$

The second part of the potential energy comes from the brane world correction to gravity which in the RandallSundrum scenario is given by,

$$
U_{B}=-\frac{G l_{s}^{2} m_{1} m_{2}}{\left|\vec{r}-\overrightarrow{r^{\prime}}\right|^{3}}
$$


The brane world correction to the potential at point $\vec{r}$ due to a point mass $m$ at the point $\overrightarrow{r^{\prime}}$ is

$$
\Phi_{B}(\vec{r})=-\frac{G l_{s}^{2} m}{\left|\vec{r}-\overrightarrow{r^{\prime}}\right|^{3}}
$$

For a mass distribution with density $\rho\left(\overrightarrow{r^{\prime}}\right)$, the formulas above take the following forms,

$$
\begin{gathered}
\Phi_{N}(\vec{r})=-G \int \frac{\rho\left(\overrightarrow{r^{\prime}}\right) d^{3} \overrightarrow{r^{\prime}}}{\left|\vec{r}-\overrightarrow{r^{\prime}}\right|} \\
\Phi_{B}(\vec{r})=-G l_{s}^{2} \int \frac{\rho\left(\overrightarrow{r^{\prime}}\right) d^{3} \overrightarrow{r^{\prime}}}{\left|\vec{r}-\overrightarrow{r^{\prime}}\right|^{3}} \\
U_{N}=-G \iint \frac{\rho(\vec{r}) \rho\left(\overrightarrow{r^{\prime}}\right) d^{3} \vec{r} d^{3} \overrightarrow{r^{\prime}}}{\left|\vec{r}-\overrightarrow{r^{\prime}}\right|}=\int \Phi_{N}(\vec{r}) \rho(\vec{r}) d^{3} \vec{r} \\
U_{B}=-G l_{s}^{2} \iint \frac{\rho(\vec{r}) \rho\left(\overrightarrow{r^{\prime}}\right) d^{3} \vec{r} d^{3} \overrightarrow{r^{\prime}}}{\left|\vec{r}-\overrightarrow{r^{\prime}}\right|^{3}}=\int \Phi_{B}(\vec{r}) \rho(\vec{r}) d^{3} \vec{r}
\end{gathered}
$$

\section{A. Newtonian Potential and Potential Energy for a spherically symmetric ball of constant density}

Let us consider a spherically symmetric ball of radius $R$ and of constant density $\rho$.In spherical co-ordinate system the formula for $\Phi_{N}(r)$ gives,

$$
\Phi_{N}(r)=-G \rho \iiint \frac{r^{2} d r^{\prime} \sin \theta d \theta d \phi}{\left(r^{2}+r^{\prime 2}-2 r r^{\prime} \cos \theta\right)^{1 / 2}}
$$

In the expression above, the integral over $\phi$ ranges from 0 to $2 \pi$ and for $\theta$, it ranges from 0 to $\pi$. The integral over $\phi$ gives a factor of $2 \pi$. The integral over $\theta$ can be carried out by making the following change of variable

$$
\xi=r^{2}+r^{\prime 2}-2 r r^{\prime} \cos \theta ; d \xi=2 r r^{\prime} \sin \theta d \theta
$$

The integration over $\xi$ ranges from $\xi=\left(r-r^{\prime}\right)^{2}$ to $\xi=\left(r+r^{\prime}\right)^{2} \quad$ After carrying out integrations over $\phi$ and $\theta$, we obtain

$$
\Phi_{N}(r)=-2 \pi G \rho \int\left[\left(r+r^{\prime}\right)-\left|r-r^{\prime}\right|\right] \frac{r^{\prime}}{r} d r^{\prime}
$$

For $r>a\left(>r^{\prime}\right)$, and $0 \leq r^{\prime} \leq a$

$$
\Phi_{N}(r)=-2 \pi G \rho \int_{0}^{a}\left[\left(r+r^{\prime}\right)-\left(r-r^{\prime}\right)\right] \frac{r^{\prime}}{r} d r^{\prime}=-4 \pi \frac{G \rho}{r} \int_{0}^{a} r^{\prime 2} d r^{\prime}=-\frac{4 \pi a^{3} \rho G}{3 r}=-\frac{G M}{r}
$$

For $r<b\left(<r^{\prime}\right)$, and $b \leq r^{\prime} \leq R$

$$
\Phi_{N}(r)=-2 \pi G \rho \int_{0}^{a}\left[\left(r+r^{\prime}\right)-\left(r^{\prime}-r\right)\right] \frac{r^{\prime}}{r} d r^{\prime}=-4 \pi G \rho \int_{b}^{R} r^{\prime} d r^{\prime}=-2 \pi G \rho\left(R^{2}-b^{2}\right)
$$

which is constant independent of $r$. Since force is given by the gradient of the potential with respect to $r$, $F=$ $-\operatorname{grad}_{r}\left[\Phi_{N}(r)\right]$, there is no gravitational force within the spherical shell. Once we know the potential , it is easy to calculate the potential energy. It is given by,

$$
U_{N}=-G \iint \frac{\rho(\vec{r}) \rho\left(\overrightarrow{r^{\prime}}\right) d^{3} \vec{r} d^{3} \overrightarrow{r^{\prime}}}{\left|\vec{r}-\overrightarrow{r^{\prime}}\right|}=\int \Phi_{N}(\vec{r}) \rho(\vec{r}) d^{3} \vec{r}
$$


Let us consider the case when $r>r^{\prime}$. Taking $a=r-\epsilon$, we obtain

$$
\Phi_{N}(r)=-\frac{4 \pi G \rho(r-\epsilon)^{3}}{3 r}
$$

The potential energy is given by,

$$
U_{N}==\int_{(|\vec{r}| \leq R)} \Phi_{N}(\vec{r}) \rho(\vec{r}) d^{3} \vec{r}
$$

Substituting the expression for $\Phi_{N}(r)$, we obtain,

$$
U_{N}=-\int_{\epsilon}^{R} \frac{4 \pi G \rho(r-\epsilon)^{3}}{3 r} \times 4 \pi \rho r^{2} d r
$$

This integral above is convergent, and therefore, we can take the limit $\epsilon \rightarrow 0$ before evaluating the integral.After integrating the resulting expression, we obtain

$$
U_{N}=-\frac{16 \pi^{2} G \rho^{2} R^{5}}{15}=-\frac{3}{5} \times \frac{G M^{2}}{R}
$$

\section{B. Potential and Potential Energy for a spherically symmetric ball of constant density due to Randall-Sundrum Brane world correction to Gravity}

The potential and the potential energy due to the brane world correction term is calculated exactly in the same way as in the case of Newtonian gravity.However, in this case there is a new element. The outer shell exerts force on the inner shells, and therefore, the effect of outer shell on the inner shell can not be neglected.

\section{The Potential at a distance from a spherically symmetric ball}

The potential $\Phi_{B}(r)$ in spherical co-ordinate system is given by,

$$
\Phi_{B}(r)=-G l_{s}^{2} \rho \iiint \frac{r^{\prime 2} d r^{\prime} \sin \theta d \theta d \phi}{\left(r^{2}+r^{\prime 2}-2 r r^{\prime} \cos \theta\right)^{3 / 2}}
$$

As in the previous section, the integral over $\phi$ ranges from 0 to $2 \pi$ and the integral over $\theta$ ranges from 0 to $\pi$. Integral over $\phi$ gives factor of $2 \pi$. Integral over $\theta$ can be carried out by making the following change of variable

$$
\xi=r^{2}+r^{2}-2 r r^{\prime} \cos \theta ; \quad d \xi=2 r r^{\prime} \sin \theta d \theta ;
$$

The integration over $\xi$ ranges from $\xi=\left(r-r^{\prime}\right)^{2}$ to $\xi=\left(r+r^{\prime}\right)^{2} \quad$ After carrying out integrations over $\phi$ and $\theta$, we obtain

$$
\Phi_{B}(r)=-2 \pi G l_{s}^{2} \rho \int\left[\frac{1}{\left|r-r^{\prime}\right|}-\frac{1}{r+r^{\prime}}\right] \frac{r^{\prime}}{r} d r^{\prime}
$$

For $r>a\left(>r^{\prime}\right)$, and $0 \leq r^{\prime} \leq a$

$$
\Phi_{B}(r)=-2 \pi G l_{s}^{2} \rho \int_{0}^{a}\left[\frac{1}{r-r^{\prime}}-\frac{1}{r+r^{\prime}}\right] \frac{r^{\prime}}{r} d r^{\prime}
$$

After some algebraic manipulations, this integral can be evaluated.The result is

$$
\Phi_{B}(r)=-2 \pi G l_{s}^{2} \rho\left[\ln \frac{r+a}{r-a}-\frac{2 a}{r}\right]
$$

Large distance limit of the potential can be evaluated in two different ways. From the formula,

$$
\Phi_{B}(\vec{r})=-G l_{s}^{2} \int_{0}^{a} \frac{\rho\left(\overrightarrow{r^{\prime}}\right) d^{3} \vec{r}^{\prime}}{\left|\vec{r}-\overrightarrow{r^{\prime}}\right|^{3}}
$$


we find that for $r>>a$,

$$
\Phi_{B}(\vec{r})=-\frac{G l_{s}^{2}}{|\vec{r}|^{3}} \int_{0}^{a} \rho\left(\overrightarrow{r^{\prime}}\right) d^{3} \vec{r}^{\prime}=-\frac{4 \pi G l_{s}^{2} \rho a^{3}}{3 r^{3}}=-\frac{G l_{s}^{2} M}{r^{3}}
$$

The other way is to consider the formula,

$$
\Phi_{B}(r)=-2 \pi G l_{s}^{2} \rho\left[\ln \frac{r+a}{r-a}-\frac{2 a}{r}\right]
$$

which has been derived for $r>a$, and therefore, can also be written as,

$$
\Phi_{B}(r)=-2 \pi G l_{s}^{2} \rho\left[\ln \frac{1+a / r}{1-a / r}-\frac{2 a}{r}\right]=-2 \pi G l_{s}^{2} \rho\left[\ln (1+a / r)-\ln (1-a / r)-\frac{2 a}{r}\right]
$$

Replacing the logarithms above by their series expansion, after some algebraic manipulations we obtain,

$$
\Phi_{B}(r)=-\frac{4 \pi G l_{s}^{2} \rho a^{3}}{3 r^{3}}-\frac{4 \pi G l_{s}^{2} \rho a^{5}}{5 r^{5}}-\frac{4 \pi G l_{s}^{2} \rho a^{7}}{7 r^{7}}-\ldots=-\frac{G l_{s}^{2} M}{r^{3}}-\ldots \ldots \ldots
$$

\section{Force at a distance from a spherically symmetric ball}

Force at a distance from a spherically symmetric ball can be calculated by evaluating the gradient of the potential, $F=-\operatorname{Grad} \Phi_{B}(r)=-\frac{d \Phi_{B}(r)}{d r}$. The potential is given by,

$$
\Phi_{B}(r)=-2 \pi G l_{s}^{2} \rho\left[\ln \frac{r+a}{r-a}-\frac{2 a}{r}\right]
$$

and therefore, the force is given by

$$
F=2 \pi G l_{s}^{2} \rho\left[\frac{1}{r+a}-\frac{1}{r-a}+\frac{2 a}{r^{2}}\right]=-4 \pi a G l_{s}^{2} \rho\left[\frac{1}{r^{2}-a^{2}}-\frac{1}{r^{2}}\right]
$$

Let us calculate the force at a very small distance from the surface. We take $r-a=\epsilon, \epsilon$ very small, and substitute it in the equation above.After some algebraic manipulation we obtain,

$$
F=2 \pi G l_{s}^{2} \rho\left[-\frac{1}{\epsilon}+\frac{2}{a}\right]
$$

This is clearly a skin effect. However, this force is not as strong as one may think. At a distance of $1 \mathrm{~mm}$ from the surface of a neutron star, this force is weaker than the force of Newtonian gravity.The reason is that this force depends on the density and not the mass. However, this force can be large close to the surface of a small spherical grain and may even overtake the force due to Newtonian gravity.

\section{Potential inside a Spherical Shell}

Let us consider a spherical shell of outer radius $R$ and inner radius $b$. At a point $r$ inside the shell, the potential is

$$
\Phi_{B}^{\prime}(r)=-2 \pi G l_{s}^{2} \rho \int_{b}^{R}\left[\frac{1}{\left|r-r^{\prime}\right|}-\frac{1}{r+r^{\prime}}\right] \frac{r^{\prime}}{r} d r^{\prime}
$$

Since $r<b \leq r^{\prime} \leq R$, the formula above becomes,

$$
\Phi_{B}^{\prime}(r)=-2 \pi G l_{s}^{2} \rho \int_{b}^{R}\left[\frac{1}{r^{\prime}-r}-\frac{1}{r+r^{\prime}}\right] \frac{r^{\prime}}{r} d r^{\prime}=-2 \pi G l_{s}^{2} \rho \int_{b}^{R} \frac{2 r^{\prime} d^{\prime}}{r^{\prime 2}-r^{2}}=-2 \pi G l_{s}^{2} \rho \ln \frac{R^{2}-r^{2}}{b^{2}-r^{2}}
$$




\section{Force inside the shell}

Force inside the shell can be calculated by taking the gradient of the potential, $F=-G r a d\left[\Phi_{B}^{\prime}(r)\right]=-\frac{d \Phi_{B}^{\prime}(r)}{d r}$. For the potential,

$$
\Phi_{B}^{\prime}(r)=-2 \pi G l_{s}^{2} \rho \ln \frac{R^{2}-r^{2}}{b^{2}-r^{2}}
$$

we obtain,

$$
F(r)=2 \pi G l_{s}^{2} \rho\left[\frac{2 r}{b^{2}-r^{2}}-\frac{2 r}{R^{2}-r^{2}}\right]
$$

From the expression above it is obvious that $F(r)>0$. This means that it is directed in the direction in which $r$ increases.In other words, it is directed towards the inner boundary surface of the shell. At the centre of the shell, $r=0$, and therefore, the force is zero.But any small perturbation will move the test particle away towards the inner boundary surface of the shell. This is a new element that emerges from the brane world correction to Newton's law of gravity. We know that the force due to Newtonian gravity is zero inside a spherically symmetric shell. If one can find the above mentioned effect inside a shell, it will provide a very clean test of brane world corrections to Newtonian gravity. May be such experiments can be carried out on board artificial satellites.

In this case, as in the case of spherical ball, there is skin effect. The force of attraction close to the inner boundary of the shell becomes large. This can be seen as follows. Let $b=r+\epsilon$. This simply means that the layer at $r$ is just bellow the inner boundary of the shell ( $\epsilon$ distance away from the inner surface).

In this case the force at $r$ is given by,

$$
F=2 \pi G l_{s}^{2} \rho\left[\frac{1}{\epsilon}-\frac{2 r}{R^{2}-r^{2}}\right]
$$

\section{Cancellation of the Skin Effect on the Inner Layers of a spherical solid ball}

Any interior layer of the spherical ball experiences two types of forces: one is due to a sphere just bellow it and other is due to the spherical shell outside. These two types of forces give rise to a large force on this layer due to the skin effect. Remarkably, these two forces act in opposite directions and the forces due to skin effect are equal in magnitude and exactly cancel each other.It is easy to verify this. On the layer at $r$, let $F_{1}$ be the force due the inner sphere and $F_{2}$ be the force due to the outer shell.

$$
\begin{gathered}
F_{1}=2 \pi G l_{s}^{2} \rho\left[-\frac{1}{\epsilon}+\frac{2}{r}\right] \\
F_{2}=2 \pi G l_{s}^{2} \rho\left[\frac{1}{\epsilon}-\frac{2 r}{R^{2}-r^{2}}\right]
\end{gathered}
$$

Therefore, the net force at any point on a layer at $r$, is given by

$$
F=F_{1}+F_{2}=2 \pi G l_{s}^{2} \rho\left[\frac{2}{r}-\frac{2 r}{R^{2}-r^{2}}\right]
$$

This result is valid for every layer except the one at the outer most boundary. For the outer most layer, there is an inner sphere but no outer shell. There is no cancellation of the skin effect on this layer. Therefore, it experiences a large force. This will give rise to effect similar to surface tension in normal liquids.

\section{The Potential Energy}

We found in the previous sections that, for a sphere ball, the potential on a given layer at a radial distance $r$ from the centre consists of two parts.First part is due to a interior spherical ball. It is given by,

$$
\Phi_{B}(r)=-2 \pi G l_{s}^{2} \rho\left[\ln \frac{r+a}{r-a}-\frac{2 a}{r}\right]
$$


The second part is due to the outer spherical shell. It is given by,

$$
\Phi_{B}^{\prime}(r)=-2 \pi G l_{s}^{2} \rho \ln \frac{R^{2}-r^{2}}{b^{2}-r^{2}}
$$

The potential energy also consists of two parts. The first part of the potential energy is obtained by taking the radius of inner sphere $a=r-\epsilon$ in the formula for the potential, multiplying the potential by $4 \pi r^{2} d r \rho$, the mass of the thin layer and then integrating the resulting expression from $r=\epsilon$ to $r=R$, where $\epsilon$ is the smallest inter particle distance, $R$ is the total radius of the spherical ball. This is given by,

$$
U_{1}=-2 \pi G l_{s}^{2} \rho \int_{\epsilon}^{R}\left[\ln \frac{2 r-\epsilon}{\epsilon}-\frac{2(r-\epsilon)}{r}\right] 4 \pi \rho r^{2} d r
$$

The second part of the potential energy is obtained by taking the inner radius of the spherical shell, $b=r+\epsilon$ in the formula for the potential due to the shell, multiplying the potential by $4 \pi r^{2} d r \rho$, the mass of the thin layer, and then integrating the resulting expression from $r=0$ to $r=R$, where $R$ is the total radius of the spherical ball. This is given by,

$$
U_{2}=-2 \pi G l_{s}^{2} \rho \int_{0}^{R}\left[\ln \frac{R^{2}-r^{2}}{(r+\epsilon)^{2}-r^{2}}\right] 4 \pi \rho r^{2} d r
$$

After some algebraic manipulations, we obtain

$$
\begin{aligned}
& U_{2}=-2 \pi G l_{s}^{2} \rho \int_{0}^{R}\left[\ln \frac{R+r}{\epsilon}+\ln \frac{R-r}{\epsilon}\right. \\
& \left.-\ln \frac{2 r+\epsilon}{\epsilon}\right] 4 \pi \rho r^{2} d r
\end{aligned}
$$

The total potential energy is given by,

$$
\begin{aligned}
U= & U_{1}+U_{2} \\
= & -8 \pi^{2} G l_{s}^{2} \rho^{2}\left[\int_{\epsilon}^{R} r^{2} d r\left(\ln \frac{2 r-\epsilon}{\epsilon}-\frac{2(r-\epsilon)}{r}\right)\right. \\
& +\int_{0}^{R} r^{2} d r\left(\ln \frac{R+r}{\epsilon}+\right. \\
& \left.\left.\ln \frac{R-r}{\epsilon}-\ln \frac{2 r+\epsilon}{\epsilon}\right)\right]
\end{aligned}
$$

:In the $\epsilon \rightarrow 0$ limit, it is not difficult to show that

$$
\int_{\epsilon}^{R} \ln \frac{2 r-\epsilon}{\epsilon} r^{2} d r-\int_{0}^{R} \ln \frac{2 r+\epsilon}{\epsilon} r^{2} d r \quad \rightarrow 0
$$

Therefore, the potential energy is given by,

$$
U=-8 \pi^{2} G l_{s}^{2} \rho^{2}\left[\int_{\epsilon}^{R} r^{2} d r\left(-\frac{2(r-\epsilon)}{r}\right)+\int_{0}^{R} r^{2} d r\left(\ln \frac{R+r}{\epsilon}+\ln \frac{R-r}{\epsilon}\right)\right]
$$

The last two integrals after change of variables amounts to evaluating standard table integrals of the form $\int x^{n} \ln x$. We simply write down the results.

$$
\begin{gathered}
\int_{0}^{R} \ln \frac{R+r}{\epsilon} r^{2} d r=\frac{1}{3} R^{3} \ln \frac{R}{\epsilon}+\frac{2}{3} R^{3} \ln 2-\frac{5}{18} R^{3} \\
\int_{0}^{R} \ln \frac{R-r}{\epsilon} r^{2} d r=\frac{1}{3} R^{3} \ln \frac{R}{\epsilon}-\frac{11}{18} R^{3} \\
\int_{\epsilon}^{R} r^{2} d r\left(-\frac{2(r-\epsilon)}{r}\right)=-\frac{2}{3} R^{3}
\end{gathered}
$$


Therefore,

$$
\begin{aligned}
U & =-8 \pi^{2} G l_{s}^{2} \rho^{2}\left[\frac{2}{3} R^{3} \ln \frac{R}{\epsilon}+\frac{2}{3} R^{3} \ln 2-\frac{14}{9} R^{3}\right]=-8 \pi^{2} G l_{s}^{2} \rho^{2}\left[\frac{2}{3} R^{3} \ln \frac{R}{\epsilon}-\frac{2}{3} R^{3} \ln (5.16)\right] \\
& =-8 \pi^{2} G l_{s}^{2} \rho^{2}\left[\frac{2}{3} R^{3} \ln \left(\frac{R}{5.16 \epsilon}\right)\right]
\end{aligned}
$$

With a redefined $\epsilon$, we can write it as,

$$
U=-\frac{16 \pi^{2}}{3} G l_{s}^{2} \rho^{2} R^{3} \ln \frac{R}{\epsilon}=-\frac{3 G M^{2} l_{s}^{2}}{R^{3}} \ln \frac{R}{\epsilon}
$$

If $N$ is the number of particles in the spherical ball and $m$ is the mass of the elementary constituent particles, then $M=N m$ and we can write the total potential energy due to Randall-Sundrum brane-world induced correction term as

$$
U=U_{R S}=-\frac{3 G N^{2} m^{2} l_{s}^{2}}{R^{3}} \ln \frac{R}{\epsilon}
$$

The small parameter, $\epsilon$, in the formulae above should be interpreted as the minimum inter-particle distance in the spherically symmetric volume.

\section{APPENDIX-II}

We want to calculate the extrema of the energy functionals considered in the main text of the paper. We will consider only the white dwarf stars. The results for neutron stars can be obtained simply by replacing the electron mass by the neutron mass.

Most literature on white dwarf and neutron stars treat the ultra-relativistic limit of such stars taking the kinetic energy term as, K.E. $=N c|p|$. We will first show that in the presence of brane corrections such systems do not have any stable quantum mechanical ground state. In this case the total energy including the brane world corrections as a function of $R$, is given by

$$
E(R)=N c|p|-\frac{3}{5} \times \frac{G m_{n}^{2} N^{2}}{R}-\frac{3 G m_{n}^{2} N^{2} l_{s}^{2}}{R^{3}} \times \ln \frac{R}{\epsilon}
$$

Substituting $p=\frac{\hbar N^{1 / 3}}{R}$, we obtain

$$
E(R)=\frac{c \hbar N^{4 / 3}}{R}-\frac{3}{5} \times \frac{G m_{n}^{2} N^{2}}{R}-\frac{3 G m_{n}^{2} N^{2} l_{s}^{2}}{R^{3}} \times \ln \frac{R}{\epsilon}
$$

With $N_{\max }$ and $\alpha$, defined as

$$
N_{\max }=\frac{5}{3} \times \frac{c \hbar}{G m_{n}^{2}} \quad ; \quad \alpha=\left(N / N_{\max }\right)^{2 / 3}
$$

we can write the total energy given above as

$$
E(R)=c \hbar N^{4 / 3}\left[\frac{1}{R}-\frac{\alpha}{R}-\frac{15 \alpha l_{s}^{2}}{R^{3}} \times \ln \frac{R}{\epsilon}\right]
$$

The extremum is obtained by equating the the derivative of $E(R)$ with respect to $R$ to zero with $N$ held fixed.

$$
\frac{d E}{d R}=-\frac{c \hbar N^{4 / 3}}{R^{2}}\left(1-\alpha-\frac{45 \alpha l_{s}^{2}}{R^{2}} \times \ln \frac{R}{\epsilon_{1}}\right)=0
$$

where $\epsilon_{1}=\gamma \epsilon ; \quad \gamma$ being some positive number, $1<\gamma<10$. To find the nature of the extremum, we calculate the second derivative of $E(R)$ with respect to $R$.

$$
\frac{d^{2} E}{d R^{2}}=\frac{2 c \hbar N^{4 / 3}}{R^{3}}\left(1-\alpha-\frac{45 \alpha l_{s}^{2}}{R^{2}} \times \ln \frac{R}{\epsilon_{1}}\right)-\frac{c \hbar N^{4 / 3}}{R^{2}}\left(\frac{90 \alpha l_{s}^{2}}{R^{3}} \times \ln \frac{R}{\epsilon_{2}}\right)
$$


where $\epsilon_{2}=\gamma_{1} \epsilon_{1} ; \quad \gamma_{1}$ being some positive number, $1<\gamma_{1}<10$. The quantities $\epsilon, \epsilon_{1}$ and $\epsilon_{2}$ as an order of magnitude can be taken to be equal to $\frac{R}{N^{1 / 3}}$. In the equation above, the first term is zero from the condition that $\frac{d E}{d R}=0$. The second term is strictly negative. Therefore, the extremum of the function, $E(R)$, is a maximum. The energy $E(R)$ as a function of $R$, starts from $-\infty$ at $R=0$, rises very sharply to a positive value and develops a maximum very close to $R=0$, and then from the positive side of energy it slowly approaches zero at $R=\infty$.

Next we consider the total energy functional given by

$$
E(R)=K_{N}+U_{N}(R)=N c\left(p^{2}+m_{e}^{2} c^{2}\right)^{1 / 2}-N m_{e} c^{2}-\frac{3}{5} \times \frac{G m_{n}^{2} N^{2}}{R}
$$

Substituting $p=\frac{\hbar N^{1 / 3}}{R}$, we obtain

$$
E(R)=N c\left(\frac{\hbar^{2} N^{2 / 3}}{R^{2}}+m_{e}^{2} c^{2}\right)^{1 / 2}-N m_{e} c^{2}-\frac{3}{5} \times \frac{G m_{n}^{2} N^{2}}{R}
$$

The extremum with respect to $R$ is given by the condition,

$$
\frac{d E}{d R}=-\frac{c \hbar N^{4 / 3}}{R^{2}}\left[\frac{N^{1 / 3} \hbar}{\left(\hbar^{2} N^{2 / 3}+m_{e}^{2} c^{2} R^{2}\right)^{1 / 2}}-\frac{3}{5} \times \frac{G m_{n}^{2} N^{2 / 3}}{c \hbar}\right]=0
$$

In terms of the parameters, $R_{0}\left(=\frac{\hbar N^{1 / 3}}{m_{e} c}\right)$ and $\alpha$, the equation above simplifies to the form

$$
\frac{d E}{d R}=-\frac{c \hbar N^{4 / 3}}{R^{2}}\left[\frac{1}{\left(1+\frac{R^{2}}{R_{0}^{2}}\right)^{1 / 2}}-\alpha\right]=0
$$

To find the nature of the extremum, we calculate the second derivative of $E(R)$ with respect to $R$. It is given by,

$$
\frac{d^{2} E}{d R^{2}}=\frac{2 c \hbar N^{4 / 3}}{R^{3}}\left[\frac{1}{\left(1+\frac{R^{2}}{R_{0}^{2}}\right)^{1 / 2}}-\alpha\right]-\frac{c \hbar N^{4 / 3}}{R^{2}}\left[\frac{-R}{R_{0}^{2}\left(1+\frac{R^{2}}{R_{0}^{2}}\right)^{3 / 2}}\right]
$$

The first term is zero because of the condition, $\frac{d E}{d R}=0$. Therefore,

$$
\frac{d^{2} E}{d R^{2}}=\frac{c \hbar N^{4 / 3}}{R}\left[\frac{1}{R_{0}^{2}\left(1+\frac{R^{2}}{R_{0}^{2}}\right)^{3 / 2}}\right]
$$

which is strictly positive implying that the extremum is a minimum. The energy $E(R)$ as a function of $R$, starts from $\infty$ at $R=0$ and rolls down to a minimum at a negative value of $E(R)$ and then from the negative side of energy it slowly approaches zero at $R=\infty$.

Now we consider extremum of the total energy functional given by,

$$
E(R)=K_{N}+U_{N}(R)+U_{R S}=N c\left(p^{2}+m_{e}^{2} c^{2}\right)^{1 / 2}-N m_{e} c^{2}-\frac{3}{5} \times \frac{G m_{n}^{2} N^{2}}{R}-\frac{3 G l s^{2} m_{n}^{2} N^{2}}{R^{3}} \times \ln \frac{R}{\epsilon}
$$

Substituting $p=\frac{\hbar N^{1 / 3}}{R}$, we obtain

$$
E(R)=N c\left(\frac{\hbar^{2} N^{2 / 3}}{R^{2}}+m_{e}^{2} c^{2}\right)^{1 / 2}-N m_{e} c^{2}-\frac{3}{5} \times \frac{G m_{n}^{2} N^{2}}{R}-\frac{3 G l s^{2} m_{n}^{2} N^{2}}{R^{3}} \times \ln \frac{R}{\epsilon}
$$

The necessary condition for the extremum is given by,

$$
\frac{d E(R)}{d R}=-\frac{c N^{5 / 3} \hbar^{2}}{R^{3}\left(\frac{\hbar^{2} N^{2 / 3}}{R^{2}}+m_{e}^{2} c^{2}\right)^{1 / 2}}+\frac{3}{5} \times \frac{G m_{n}^{2} N^{2}}{R^{2}}+\frac{9 G l s^{2} m_{n}^{2} N^{2}}{R^{4}} \times \ln \frac{R}{\epsilon}-\frac{3 G l s^{2} m_{n}^{2} N^{2}}{R^{4}}=0
$$

The last term, in the equation above, can be absorbed in the third term containing the logarithm by simply redefining the parameter $\epsilon$. As before, we denote it by $\epsilon_{1}$. With the redefined $\epsilon$, the equation above takes the following form,

$$
\frac{d E}{d R}=-\frac{c \hbar N^{4 / 3}}{R^{2}}\left[\frac{N^{1 / 3} \hbar}{\left(\hbar^{2} N^{2 / 3}+m_{e}^{2} c^{2} R^{2}\right)^{1 / 2}}-\frac{3}{5} \times \frac{G m_{n}^{2} N^{2 / 3}}{c \hbar}-\frac{9 G l s^{2} m_{n}^{2} N^{2 / 3}}{R^{2} c \hbar} \times \ln \frac{R}{\epsilon_{1}}\right]=0
$$


In terms of the parameters, $R_{0}$ and $\alpha$, the equation above simplifies to the form

$$
\frac{d E}{d R}=-\frac{c \hbar N^{4 / 3}}{R^{2}}\left[\frac{1}{\left(1+\frac{R^{2}}{R_{0}^{2}}\right)^{1 / 2}}-\alpha-\frac{15 \alpha l_{s}^{2}}{R^{2}} \times \ln \frac{R}{\epsilon_{1}}\right]=0
$$

In the main text of the paper, it is shown that

$$
\frac{R^{2}}{R_{0}^{2}}=\frac{1}{\alpha^{2}}-1+\mathcal{O}(\beta)
$$

solves the equation above. The parameter $\beta$ is defined in the text as,

$$
\beta=\frac{15 l_{s}^{2}}{R_{0}^{2}} \times \ln \frac{R}{\epsilon} \approx \frac{15 l_{s}^{2}}{R_{0}^{2}} \times \ln N^{1 / 3}
$$

For $l_{s}=1 \mathrm{~mm}$ and $N \approx 10^{57}$, we find that $\beta \approx \frac{3}{R_{0}^{2}}$. Therefore, the solution of the equation for the extremum can also be written as

$$
\frac{R^{2}}{R_{0}^{2}}=\frac{1}{\alpha^{2}}-1+\mathcal{O}\left(\frac{1}{R_{0}^{2}}\right)
$$

To make the brane world parameter dependence explicit, we can also write it as

$$
\frac{R^{2}}{R_{0}^{2}}=\frac{1}{\alpha^{2}}-1+\mathcal{O}\left(\frac{l_{s}^{2}}{R_{0}^{2}}\right)
$$

To find the nature of the extremum, we need to calculate the second derivative with respect to $R$ of the function $E(R)$. This is given by,

$$
\frac{d^{2} E}{d R^{2}}=\frac{2 c \hbar N^{4 / 3}}{R^{3}}\left[\frac{1}{\left(1+\frac{R^{2}}{R_{0}^{2}}\right)^{1 / 2}}-\alpha-\frac{15 \alpha l_{s}^{2}}{R^{2}} \times \ln \frac{R}{\epsilon_{1}}\right]-\frac{c \hbar N^{4 / 3}}{R^{2}}\left[\frac{-R}{R_{0}^{2}\left(1+\frac{R^{2}}{R_{0}^{2}}\right)^{3 / 2}}+\frac{30 \alpha l_{s}^{2}}{R^{3}} \times \ln \frac{R}{\epsilon_{2}}\right]
$$

The first term in the equation above is zero because of the condition, $\frac{d E}{d R}=0$. With $l_{s}=1 \mathrm{~mm}=0.1 \mathrm{~cm}$ and $\epsilon_{2}=\frac{R}{N^{1 / 3}}$, we can write the equation above as,

$$
\frac{d^{2} E}{d R^{2}}=\frac{c \hbar N^{4 / 3}}{R}\left[\frac{1}{R_{0}^{2}\left(1+\frac{R^{2}}{R_{0}^{2}}\right)^{3 / 2}}-\frac{0.3 \alpha \ln N^{1 / 3}}{R^{4}}\right]
$$

Using Eq.( ), this can be written as,

$$
\frac{d^{2} E}{d R^{2}}=\frac{c \hbar N^{4 / 3} \alpha^{3}}{R}\left[\frac{1}{R_{0}^{2}+\mathcal{O}\left(l_{s}^{2}\right)}-\frac{0.3 \alpha^{2} \ln N^{1 / 3}}{R_{0}^{4}\left(\alpha^{2}-1\right)^{2}+\mathcal{O}\left(l_{s}^{2} R_{0}^{2}\right)}\right]
$$

For all admissible values of the parameters for white dwarfs as well as neutron stars, the expression above is strictly positive. Therefore, the extremum is a minimum. Note that the equation for extrema is a cubic equation in $R^{2}$, and therefore, there should be two more solutions. These two solutions up to order $\beta$ are real and coincident. These two solutions are given by,

$$
\frac{R^{2}}{R_{0}^{2}}=\mathcal{O}(\beta)=\mathcal{O}\left(\frac{l_{s}^{2}}{R_{0}^{2}}\right)
$$

These solutions correspond to a maximum of the total energy $E(R)$. The energy $E(R)$ as a function of $R$, starts from $-\infty$ at $R=0$, rises very sharply to a positive value and develops a maximum very close to $R=0$, it rolls down to a minimum at a negative value of $E(R)$ and then from the negative side of energy it slowly approaches zero at $R=\infty$.

[1] L.Randall and R.Sundrum, Phys.Rev.Lett. 83, 3370 (1999) 
[2] L.Randall and R.Sundrum, Phys.Rev.Lett. 83,4690 (1999)

[3] T. Shiromizu, K. Maeda and M. Sasaki, Phys. Rev. D62,024012 (2000); M. Sasaki, T. Shiromizu and K. Maeda, Phys. Rev. D62,024008 (2000); Roy Maartens, Phys. Rev. D 62, (2000) 084023; J.M. Cline, C. Grojean and G. Servant, Phys. Rev. Lett. 834245 (1999); N. Kaloper, Phys. Rev. D 60123506 (1999); P. Binetruy, C. Deffayet, U. Ellwanger, and D. Langlois, Phys. Lett. B477, 285 (2000); E. E. Flanagan, S.-H. Tye, and I. Wasserman, Phys. Rev. D 62 044039 (2000); S. W. Hawking, T. Hertog, and H. Reall, Phys. Rev. D 62043501 (2000); S. Nojiri, S. D. Odintsov, and S. Zerbini, Phys. Rev. D 62064006 (2000); S. Nojiri and S. Odintsov, Phys. Lett. B 484, 119 (2000); L. Anchordoqui, C. Nunez, and K. Olsen, J. High Energy Phys. Phys. 10, 050 (2000); S. Nojiri and S. Odintsov, Phys. Lett. B 494, 135 (2000); S. Gubser, Phys. Rev. D 63, 084017 (2001); T. Shiromizu and D. Ida, Phys. Rev. D 64, 044015 (2001).

[4] Roy Maartens, D. Wands, B.A. Bassett, and I.P.C. Heard, Phys. Rev. D 62, 041301 (2000); E.J. Copeland, A.R. Liddle, and J.E. Lidsey, astro-ph/0006421 E.J. Copeland, A. R. Liddle and J. E. Lidsey, Phys. Rev. D 64, 023509 (2001) astro-ph/0006421; G. Huey and Lidsey, Phys. Lett. B 514,217(2001); V. Sahni, M. Sami and T. Souradeep, Phys. Rev. D65,023518(2002); A. S. Majumdar, Phys.Rev. D64 (2001) 083503; J. W. F. Valle, Astropart. Phys. 18, 287(2002) astro-ph/0111417; K. Dimopoulos, Nucl. Phys. Proc. Suppl.95, 70(2001) astro-ph/0012298; M. Sami, N. Dadhich and Tetsuya Shiromizu, Phys. Lett B 68, 118(2003) hep-th/0304187; M. Sami and V. Sahni, hep-th/0402086 M. Sami and N. Dadhich, hep-th/0405016 Shinji Tsujikawa and A. R. Liddle, JCAP 0403 (2004) 001 astro-ph/0312162.

[5] C.Germani and R.Maartens, Phys. Rev. D64 124010(2001)

[6] R.Maartens, Brane-World Gravity, gr-qc/0312059

[7] T.Wiseman, Phys. Rev. D65 124007(2002); hep-th/0111057

[8] T.Damour, Gravitation and Experiment, gr-qc/9711061

[9] E.H.Lieb et. al. The stability of matter:From atom to stars, Selecta of E.H.Lieb, Ed. W.E.Thirring, Springer-Verlag, 1991

[10] S.L.Shapiro and S.A.Teukolsky, Blacl Holes, White Dwarfs, and Neutron Stars; John Wiley and Sons, Inc, 1983

[11] J.Hartle, Physics Reports 46C 200(1978) 